\title{
Effect of wind-driven accretion on planetary migration
}

\author{
C. N. Kimmig ${ }^{1}$, C. P. Dullemond ${ }^{1}$, and W. Kley ${ }^{2}$ \\ ${ }^{1}$ Zentrum für Astronomie, Heidelberg University, Albert-Ueberle-Str. 2, 69120 Heidelberg, Germany
e-mail: mail@carolin.kim
${ }^{2}$ Institut für Astronomie und Astrophysik, Universität Tübingen, Auf der Morgenstelle 10, 72076 Tübingen, Germany
}

Received 30 July 2019 / Accepted 25 October 2019

\begin{abstract}
Context. Planetary migration is a key link between planet formation models and observed exoplanet statistics. So far, the theory of planetary migration has focused on the interaction of one or more planets with an inviscid or viscously evolving gaseous disk. Turbulent viscosity is thought to be the main driver of the secular evolution of the disk, and it is known to affect the migration process for intermediate- to high-mass planets. Recently, however, the topic of wind-driven accretion has experienced a renaissance because evidence is mounting that protoplanetary disks may be less turbulent than previously thought, and 3D non-ideal magnetohydrodynamic modeling of the wind-launching process is maturing.

Aims. We investigate how wind-driven accretion may affect planetary migration. We aim for a qualitative exploration of the main effects and not for a quantitative prediction.

Methods. We performed 2D hydrodynamic planet-disk interaction simulations with the FARGO3D code in the $(r, \phi)$ plane. The vertical coordinate in the disk and the launching of the wind are not treated explicitly. Instead, the torque caused by the wind onto the disk is treated using a simple two-parameter formula. The parameters are the wind mass-loss rate and the lever arm.

Results. We find that the wind-driven accretion process replenishes the co-orbital region in a different way than the viscous accretion process. The former always injects mass from the outer edge of the co-orbital region, and always removes mass from the inner edge, while the latter injects or removes mass from the co-orbital region depending on the radial density gradients in the disk. As a consequence, the migration behavior can differ strongly, and can under certain conditions drive rapid type-III-like outward migration. We derive an analytic expression for the parameters under which this outward migration occurs.

Conclusions. If wind-driven accretion plays a role in the secular evolution of protoplanetary disks, planetary migration studies have to include this process as well because it can strongly affect the resulting migration rate and migration direction.
\end{abstract}

Key words. protoplanetary disks - planet-disk interactions - accretion, accretion disks

\section{Introduction}

Planetary migration is an integral part of the theory of planet formation. When a planet is formed, it can migrate to vastly different radial locations through its gravitational interaction with the protoplanetary disk. As a result, the orbital elements of observed exoplanets may not reflect the location where they were born. Any model prediction of exoplanetary orbital statistics must therefore include a treatment of the migration process.

Unfortunately, the process of planetary migration is a complex affair (see, e.g., Kley \& Nelson 2012, and references therein). While the effect of the Lindblad torques is fairly well understood (Tanaka et al. 2002), the torques exerted by the gas in the co-orbital region has turned out to be hard to predict. The torques depend on the entropy gradient (Baruteau \& Masset 2008), the radiative cooling efficiency (Kley \& Crida 2008), the planet mass (Ward 1997), the viscosity of the disk (Masset 2001), and even on the motion of the planet itself (Masset \& Papaloizou 2003).

Among these effects, the role of turbulent viscosity needs particular scrutiny because doubts have been raised in recent years about whether protoplanetary disks are indeed as turbulent as they were believed to be. Evidence against strong turbulence comes from the velocity dispersion inferred from $\mathrm{CO}$ lines (Flaherty et al. 2015, 2018), the observed small scale height of the dust rings in HL Tau (Pinte et al. 2016), and from the comparison of planet-disk interaction models with recent ALMA observations (Zhang et al. 2018). Moreover, theoretical considerations about the degree of ionization of the gas in such disks have suggested that the magnetorotational instability, which is a potent driver of turbulence, may not be able to operate in the disk, except in the very upper layers (Gammie 1996). Instead, a magnetocentrifugal wind is likely to be launched (Bai \& Stone 2013), which in turn exerts a torque back onto the disk, and thus drives the gas motion in the upper layers of the disk inward, or in other words: causes accretion within the disk (Simon et al. 2013).

If wind-driven accretion becomes the new paradigm and replaces the classic viscous disk theory, then the theory of planetary migration has also to be reconsidered. In the viscous disk picture, the turbulent viscosity has a strong influence on the planetary migration (Masset 2001). When a planet opens a gap, the viscous evolution of the disk acts against this by feeding gas from both the inner and the outer disk back into the gap. The depth of the gap, and therefore the amount of material in the co-orbital (corotation) region, thus depends on the equilibrium between these two counteracting effects. Weak turbulence leads to a deep gap and a weak corotation torque. Strong turbulence, on the other hand, continues to feed gas into the corotation region, leading to a substantial corotation torque.

In contrast to the viscous disk theory, in the wind-driven accretion picture the radial inward motion of the gas in the disk 
is much more laminar. Accretion will be only inward, regardless of the radial gradients of the density, because according to the classic Blandford \& Payne (1982) model, the acceleration of the wind is driven by the injection of angular momentum into the wind. This angular momentum is extracted from the disk.

When we now insert a gap-opening planet into such a disk, the wind-driven gas motions in the disk inject mass into the gap only from the outside. At the inner edge of the gap, in stark contrast to the viscous disk model, the wind-driven accretion removes gas from the gap. In mathematical terms, the difference is that the viscous model drives accretion as a kind of diffusion process (which can transport gas both inward and outward, depending on the density gradient), while the wind-driven model drives accretion as an advection process (which transports gas only inward).

In this paper we explore how the wind-driven accretion process affects the migration of a planet. The purpose is to gain understanding, not to obtain quantitative numbers. We therefore deliberately keep the description of the wind-driven accretion process extremely simple, and focus on the gas motions in the plane of the disk.

The structure of this paper is as follows: we first describe the model assumptions, the equations we solve, and the numerical methods we employ (Sect. 2). Then we describe the setup of the simulations in Sect. 3 and show the results of a series of model calculations we performed in Sect. 4. We discuss the meaning of these results, and the caveats of the model in Sect. 5, and we conclude in Sect. 6.

\section{Model}

For the hydrodynamic simulations we used the code FARGO3D by Benítez-Llambay \& Masset (2016). We investigated the effect of the magnetocentrifugal wind in a 2D disk: radial and azimuthal. For simplification, we implemented only the effect of the magnetic wind rather than the magnetic field itself. The wind is magnetocentrifugally accelerated (Blandford \& Payne 1982), meaning that it extracts net angular momentum from the remaining material in the disk, and thus effects a radial inward drift of the gas (e.g., Ferreira 2008; Bai 2016; Suzuki et al. 2016). The resulting radial velocity $v_{r}$ can then be written as

$v_{r}=-2 r \frac{\dot{\Sigma}_{\text {wind }}}{\Sigma}(\lambda-1)$

with the convention that a negative velocity implies an inward flow. Here, $\dot{\Sigma}_{\text {wind }}$ is the mass outflow from the disk, $\Sigma$ is the surface density, and

$\lambda=\left(\frac{r_{\mathrm{A}}}{r_{\mathrm{F}}}\right)^{2}$

is the lever arm of a magnetic field, with $r_{\mathrm{A}}$ and $r_{\mathrm{F}}$ being the Alfvén point and the footpoint of the magnetic field line, respectively (see Fig. 1).

Two parameters determine the strength of the magnetic wind: the magnetic lever arm $\lambda$, and the mass outflow $\dot{\Sigma}_{\text {wind. }}$ As an assumption for the parameters, we kept the lever arm constant throughout the disk while we scaled the mass outflow rate $\dot{\Sigma}_{\text {wind }}$ proportional to the surface density,

$\dot{\Sigma}_{\text {wind }}=b \frac{\Omega_{\mathrm{K}}}{2 \pi} \Sigma$,

with $\Omega_{\mathrm{K}}$ as the Keplerian angular velocity. The mass-loss parameter $b$ was defined such that it denotes the proportional

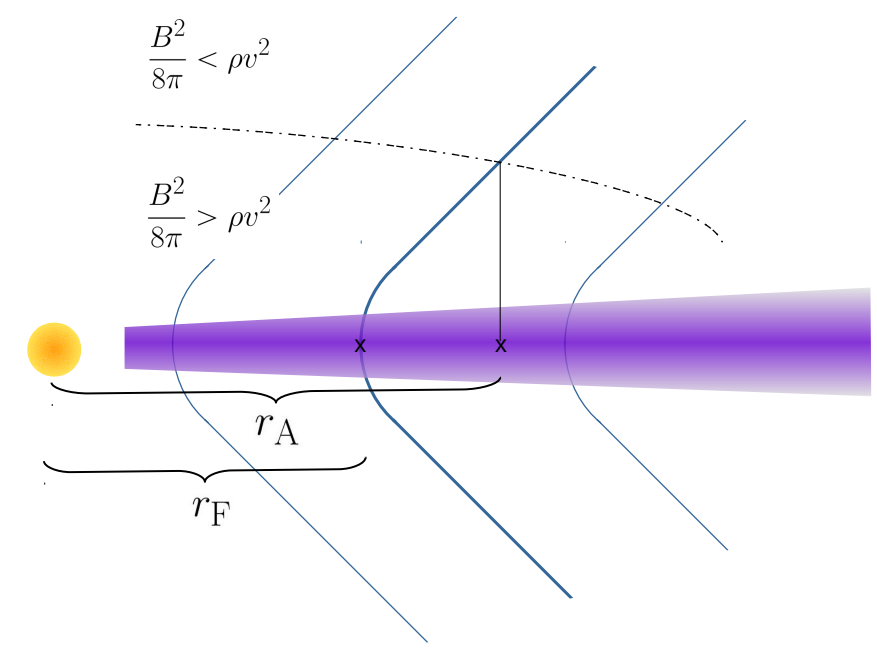

Fig. 1. Configuration of the magnetic field in the Blandford \& Payne (1982) model (schematic). The blue lines represent the magnetic field lines, the dashed line marks the Alfvén surface, and $r_{\mathrm{A}}$ and $r_{\mathrm{F}}$ represent the Alfvén point and the footpoint of the (thick) magnetic field line, respectively. Inspired by Spruit (1996)

outflow from the disk per orbital period. With this parameterization, Eq. (1) becomes

$v_{r}=-\frac{\Omega_{\mathrm{K}} r}{\pi} b(\lambda-1)$.

Hence, in our model we have $v_{r} \propto r^{-0.5}$. This is steeper than the velocity profiles in the models by Hasegawa et al. (2017) and Suzuki et al. (2016), for which the authors approximately find $v_{r} \sim r^{-0.215}$ and $v_{r} \sim r^{-0.25}$, respectively.

We implemented this inward drift as an azimuthal torque density on the disk to decelerate the gas:

$$
\begin{aligned}
\Gamma & =\dot{\Sigma}_{\text {wind }} \Omega_{\mathrm{K}} r^{2}(\lambda-1) \\
& =-\frac{\Omega_{\mathrm{K}}^{2} r^{2}}{2 \pi} b \Sigma(\lambda-1) .
\end{aligned}
$$

In addition to this torque, we added $-\dot{\Sigma}_{\text {wind }}$ as a sink term in the continuity equation.

For a steady radial flow, we require that the accretion rate of the gas within the disk remains constant. The accretion rate $\dot{M}=-2 \pi r^{2} v_{r} \Sigma$ depends on the radius $\dot{M} \propto r^{1 / 2-p}$ with the assumption of a power law for the surface density $\Sigma(r)=\Sigma_{0} \cdot r^{-p}$. Therefore, a steady-state solution is possible for $p=1 / 2$.

\section{Setup}

To illustrate the effect of the wind-driven torque on planetary migration, we placed a $M_{\text {disk }}=10^{-2} M_{\odot}$ disk around a solar mass star. The disk had a surface density profile $\Sigma \propto r^{-1 / 2}$ and spanned the radial range between 0.52 and $26 \mathrm{au}$. With this disk mass, power law and inner and outer boundary, we have $\Sigma(5.2 \mathrm{au})=70.4 \mathrm{~g} \mathrm{~cm}^{-2}$. The radial temperature structure of the disk was taken to be such that the aspect ratio of the disk is $H_{\mathrm{p}} / r=0.05$, where $H_{\mathrm{p}}=c_{\mathrm{s}} / \Omega_{\mathrm{K}}$, with $c_{\mathrm{s}}$ the isothermal sound speed. We set the turbulent viscosity parameter to $\alpha_{\text {turb }}=0$ (Shakura \& Sunyaev 1973). We inserted a planet at $r=r_{0}=5.2 \mathrm{au}$, and during the first ten orbits allowed the mass of the planet to grow linearly from 0 to the final planet mass $M_{\mathrm{p}}$. We chose two planetary masses: $M_{\mathrm{p}}=100 M_{\oplus}$ (about the mass of Saturn), and $M_{\mathrm{p}}=1 M_{\mathrm{Jup}}$. These amount to $q=M_{\mathrm{p}} / M_{*}$ 
ratios of $3 \times 10^{-4}$ and $10^{-3}$. The smoothing length of the planet potential is $s=0.6 H_{\mathrm{p}}$. The simulations with FARGO3D are dimensionless, so that the results can be scaled to other stellar masses if $q$ remains the same.

For the $(r, \phi)$ grid we chose $512 \times 656$ grid cells. The radial grid was spaced logarithmically. We ran the model for 4000 orbits at $r=r_{0}$. We employed the GPU-accelerated mode of FARGO3D. For the boundary conditions we chose the KEPLERIAN2DDENS option of FARGO3D, which is an open boundary condition where the surface density is extrapolated with a power-law slope to the ghost cells. As initial condition for the radial velocity $v_{r}$ we used Eq. (4). The initial condition for the azimuthal velocity $v_{\phi}$ was set to Kepler rotation corrected by the pressure gradient.

We varied the mass-loss parameter as $b=10^{-6}, 10^{-5}$, $10^{-4}, 10^{-3}$, and $10^{-2}$. The fiducial value of the magnetic lever arm was taken to be $\lambda=2.25$ (i.e., $r_{\mathrm{A}} / r_{\mathrm{F}}=1.5$ ). To focus on an important parameter regime, we added simulations with $b=2.15 \times 10^{-4}, 3.16 \times 10^{-4}, 4.64 \times 10^{-4}, 6.81 \times 10^{-4}$, $1.47 \times 10^{-3}$, and $3.16 \times 10^{-3}$ for the Saturn-mass planet and $b=3.16 \times 10^{-4}, 6.81 \times 10^{-4}, 1.47 \times 10^{-3}, 2.15 \times 10^{-3}$, and $3.16 \times 10^{-3}$ for the Jupiter-mass planet. We also experimented with $\lambda=9,36$, and 81 (i.e., $r_{\mathrm{A}} / r_{\mathrm{F}}=3,6$, and 9 , respectively).

For completeness, we computed the accretion rate $\dot{M}$ for our models, given our surface density profile $\Sigma(r)$ and the equation for the radial velocity (Eq. (4)):

$$
\begin{aligned}
\dot{M} & \equiv 2 \pi \Sigma r\left(-v_{r}\right)=2 \Sigma_{0} r_{0}^{0.5} \sqrt{G M_{*}} b(\lambda-1) \\
& =2.27 \times 10^{-4} b(\lambda-1) M_{\odot} \mathrm{yr}^{-1} .
\end{aligned}
$$

For $\lambda=2.25$ and $b=10^{-4}$, this yields $\dot{M}=2.8 \times 10^{-8} M_{\odot} \mathrm{yr}^{-1}$. When we compare this to the viscous disk model with $\dot{M}=3 \pi v \Sigma$, we arrive at an $\alpha$ value of $\alpha=10^{-2}$.

To test our modifications to FARGO3D, we ran a model without planet, but with a wind. For the simplified case of torque without corresponding mass loss, we tested the numerical result against the analytic solution for $v_{r}$ (Eq. (4)). We find a good match. In the other limiting case of mass loss without corresponding torque (i.e., $\lambda=1$ ), an analytic solution of an exponentially dropping surface density can be found. The numerical result matches this well also for this case.

\section{Results}

\subsection{Reference case: no wind}

In order to be able to compare our results to a reference case later on, we first ran simulations for the two different planet masses in a non-viscous disk without magnetic winds. Without viscosity and magnetic winds, the planets should not migrate.

Because of the low viscosity, the planets open a gap in the surface density (see Fig. 2). This gap formation takes longer for lower planet masses. Although the planets slightly change their semi-major axis (see Fig. 3), they do not migrate significantly without viscosity and magnetic winds.

The Saturn-mass planet $\left(M_{\mathrm{p}}=100 M_{\oplus}\right)$ migrates in the first 500 orbits, then stalls. This planet takes longer to clear a gap than the Jupiter-mass planet (Fig. 2). The gas exerts torques on the planet, and it first migrates inward. After the planet has opened a gap, the corotation and Lindblad torques are reduced. As a result, the planet slows down and comes to a halt. This halting of migration in low-viscosity disks is a known phenomenon: see, for instance, Rafikov (2002), Li et al. (2009), and Yu et al. (2010).

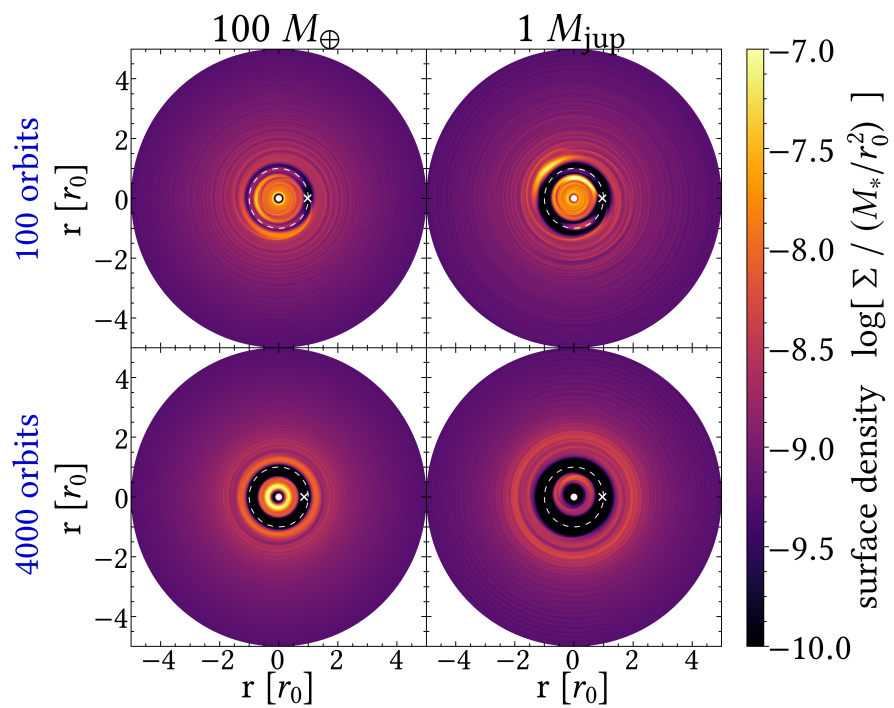

Fig. 2. Results of the inviscid reference models without wind-driven accretion: the surface density of the disk for the two different planet masses at 100 and 4000 orbits. The white cross denotes the current position of the planet, and the dashed white circle marks its initial semi-major axis at $r_{0}=5.2 \mathrm{au}$.

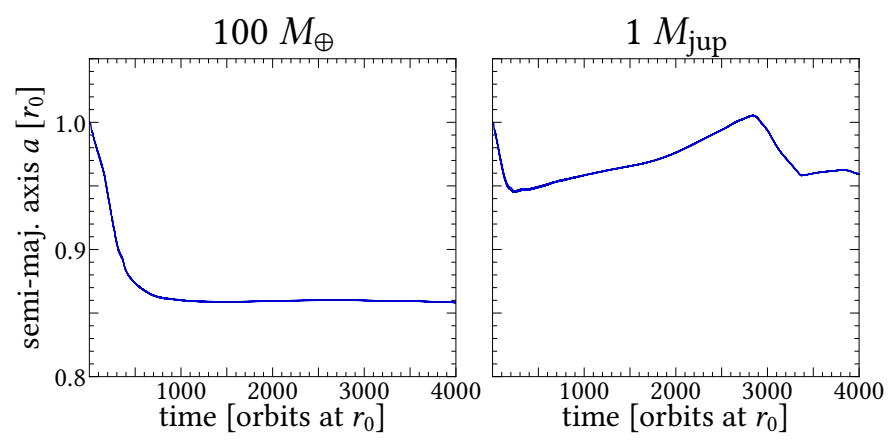

Fig. 3. Results of the inviscid reference models without wind-driven accretion: time evolution of the semi-major axes of the embedded planets.

For the Jupiter-mass planet $\left(M_{\mathrm{p}}=300 M_{\oplus}\right)$, the trend appears to be more complicated. In the first 100 orbits the planet migrates inward for the same reason as the Saturn-mass planet. It then migrates outward for a period of 2500 orbits, migrates back inward for 500 orbits, and stalls there. This migration takes place only in a small scope of $0.05 r_{0}=0.26 \mathrm{au}$. A possible explanation for this unexpected behavior is interaction with vortices that form in the disk, which are shown in Fig. 2. Such vortices were also found by Koller et al. (2003). The vortices result from perturbations from the planet in the surface density of the disk, which might be caused by the short ramp-up time of the planet mass within ten orbits. These vortices would diffuse in a viscous disk on short timescales. In the non-viscous disk, however, they remain much longer and accelerate or decelerate the planet. They therefore influence its semi-major axis. Another factor influencing its semi-major axis could be the inner disk. In the surface density (Fig. 2), the inner disk becomes eccentric due to the open inner boundary condition. The planet orbit, however, remains nearly circular: its eccentricity remains below about 0.02 . The eccentricity of the inner disk causes the planet to be located closer to the inner disk on one side than on the other side. This exerts an asymmetric torque on the planet that either 


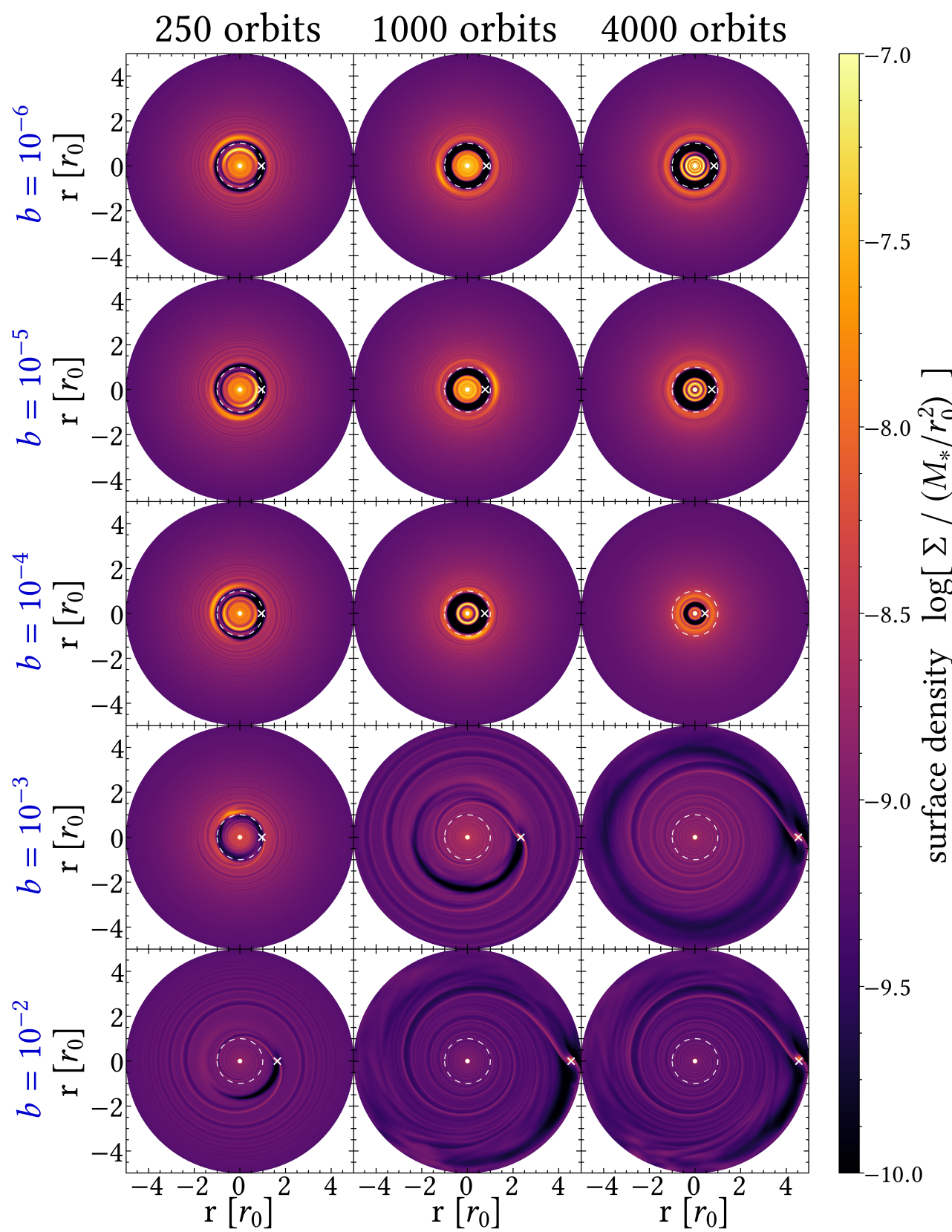

Fig. 4. Surface density maps for the wind-driven accretion case with a Saturn-mass planet. The three columns show different times. The five rows show the different values of the massloss parameter $b$. The dashed white circle marks the initial orbit of the planet. The cross denotes its current location. accelerates or decelerates the planet, causing it to migrate. The inner disk might therefore also explain the unexpected behavior of the planet.

\subsection{Model with wind-driven accretion}

We now consider wind-driven accretion. We set $\lambda=2.25$ and varied $b=10^{-6}, 10^{-5}, 10^{-4}, 10^{-3}$, and $10^{-2}$. The resulting disk surface density structures are shown for the Saturn- and Jupiter-mass planets in Figs. 4 and 5, respectively.

For low values of $b$ (cases $b=10^{-6}, 10^{-5}$, and $10^{-4}$ ), the planet opens a gap, as expected. At the outer edge of the gap, an elongated vortex forms, which is a known effect for low-viscosity disks (Koller et al. 2003; Li et al. 2000; Ataiee et al. 2013). The formation of secondary gaps in the inner disk is likewise visible, consistent with the findings of Bae et al. (2017) and Dong et al. (2017) for low-viscosity disks. These secondary rings take many orbits to form and are most pronounced in the 4000-orbit panels.
For the Saturn-mass planet models we find clear inward migration for $b \leq 10^{-4}$. The planet location (shown in the figure with a cross) after 4000 orbits is well within the original orbit (shown in the figure as the dashed circle). For the Jupiter-mass planet models the inward migration is weaker, but for the case $b=10^{-4}$, it is nevertheless clearly visible (Fig. 5).

For high values of $b$ (cases $b=10^{-3}$ and $10^{-2}$ for the Saturnmass planet and $b=10^{-2}$ for the Jupiter-mass planet) we observe an entirely different behavior. The gap is opened only partially and is asymmetric. While the co-orbital region trailing the planet is evacuated, the co-orbital region leading the planet still contains substantial amounts of gas. This leads to a positive corotation torque, which adds angular momentum to the planetary orbit. As a result, the planet rapidly migrates outward. We discuss this mechanism in more detail in Sect. 5.

In Figs. 6 and 7 we show the migration history of the planets as a function of time. For some wind strengths the Saturn-mass 


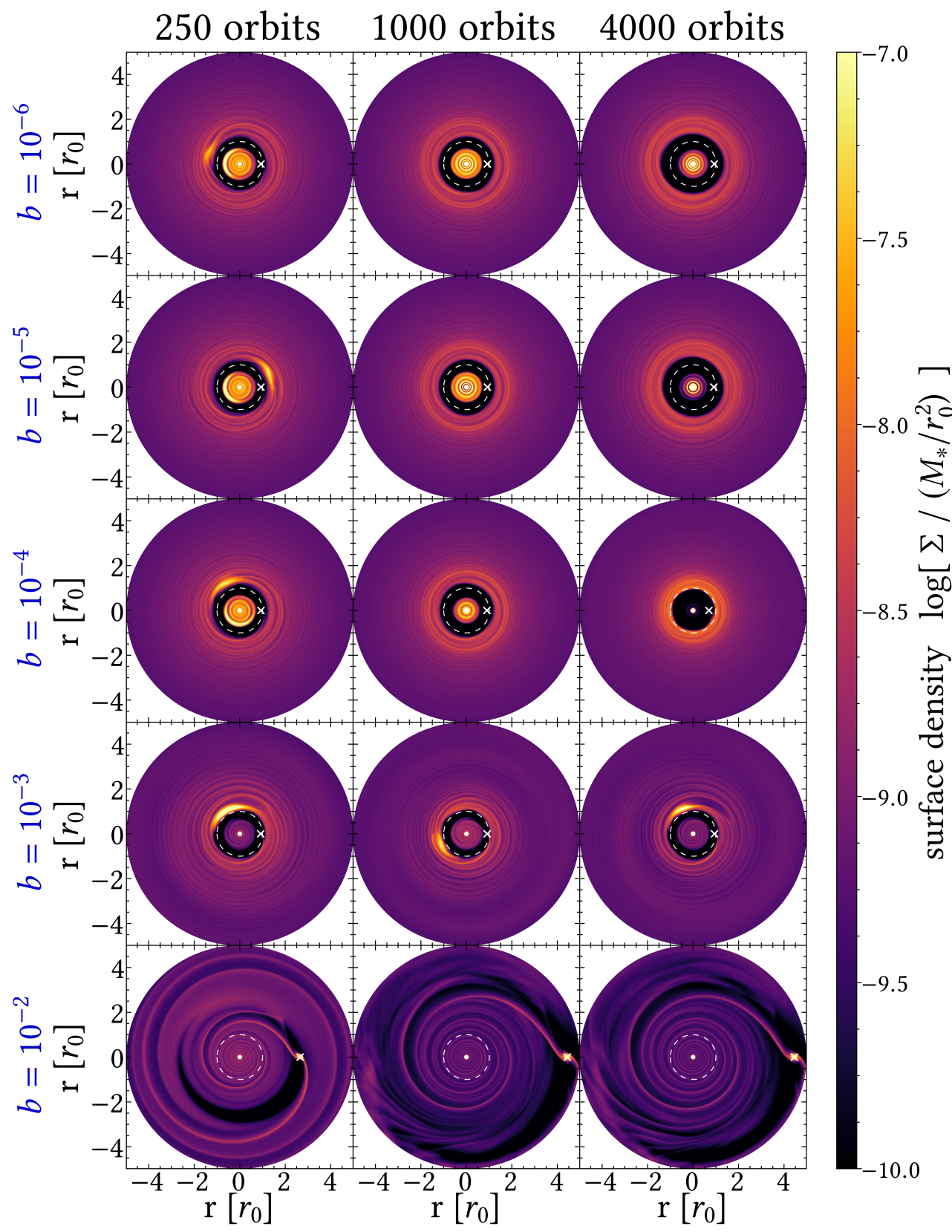

Fig. 5. Same as Fig. 4, but now for the Jupiter-mass planet. planet clearly migrates inward or outward, in comparison to the reference case. It migrates outward for $b=10^{-2}$ and $b=10^{-3}$, inward for $b=10^{-4}$, and slightly inward for $b=10^{-5}$. In the case of $b=10^{-6}$, the planet initially migrates farther than in the reference case, but stalls after 1000 orbits. In both outward-migration cases, the planet stops at $4.5 r_{0}$, which is caused by the outer boundary.

The Jupiter-mass planet migrates outward only in the case of $b=10^{-2}$, and inward for $b=10^{-4}$. In the case of $b=10^{-5}$ and $b=10^{-6}$, the planet does not migrate significantly. Particularly, it does not migrate outward as in the reference case without magnetic wind.

With a mass-loss parameter of $b=10^{-3}$, the planet performs an unexpected periodic inward and outward migration (see Fig. 7). The surface density map of this model (shown in Fig. 5 in the fourth row) shows a stronger vortex in the outer disk than in the other simulations. It does not vanish eventually, as in the other cases. We experimented with a higher resolution
$(1024 \times 1312$ grid cells $)$ and found that while the vortex remains, the oscillation vanishes, and the planet migrates somewhat farther inward. It is therefore likely that the oscillations we observe are a numerical artifact.

We suspect that this numerical artifact occurs when the simulation is set up in the transition regime between inward and outward migration. In this regime the planet is very sensitive to the different torques caused by the gas surface density, which might cause the oscillations. To test this, we performed more simulations with the same setup as before, except for the $b$ parameter. We chose a finer logarithmic sampling for $b$ to cover the transition regime for both planets, as shown in Fig. 8. We find for the Jupiter-mass planet that the setup with $b=10^{-4}$ indeed turns out to be the transition between inward and outward migration. For the Saturn-mass planet, the transition takes place at $b=3.16 \times 10^{-4}$, in which we observe no migration of the Saturn-mass planet and a slight oscillation as in the simulation with the Jupiter-mass planet. 


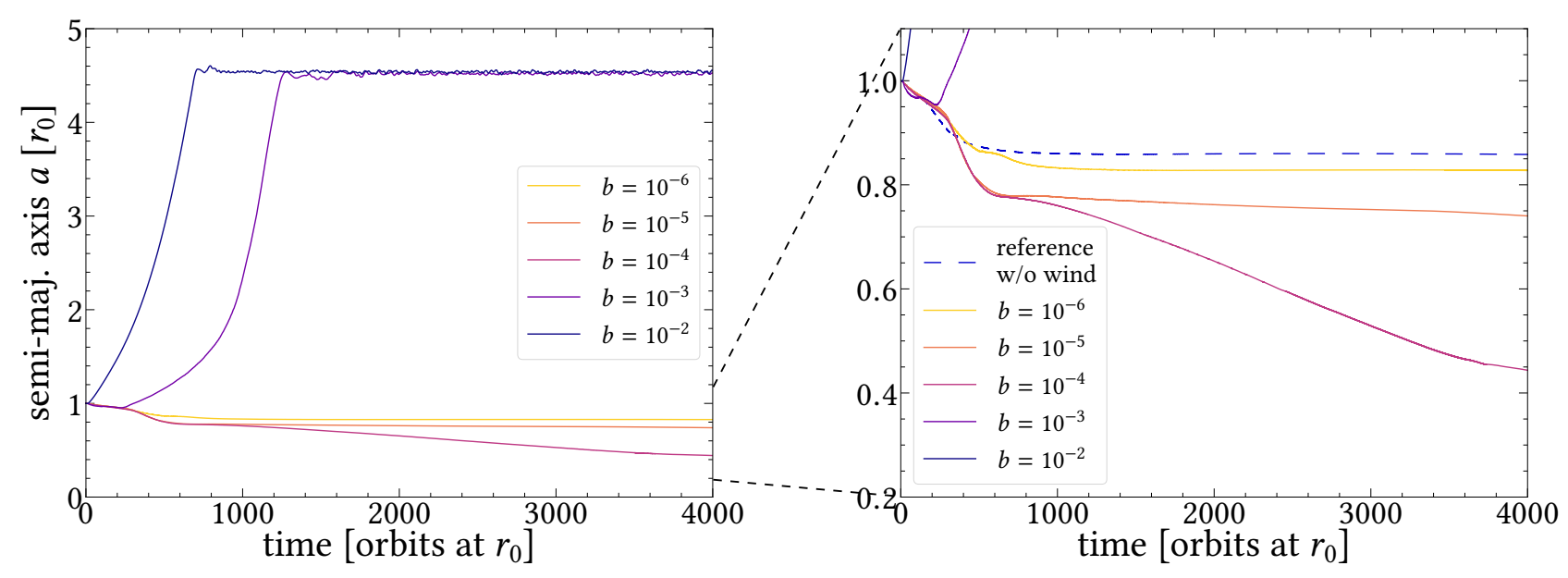

Fig. 6. Planetary migration for the wind-driven accretion case with the Saturn-mass planet for the various values of $b$. The dashed line shows the reference model without wind-driven accretion.

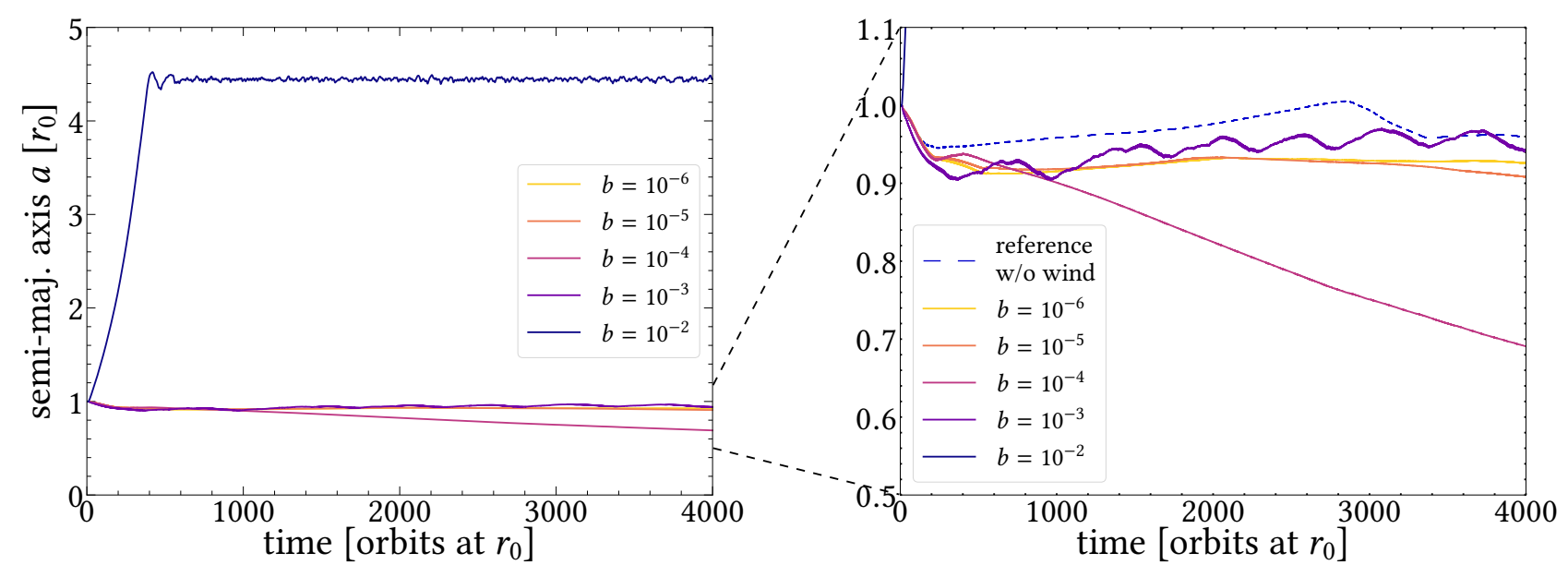

Fig. 7. Same as Fig. 6, but now for the Jupiter-mass planet.

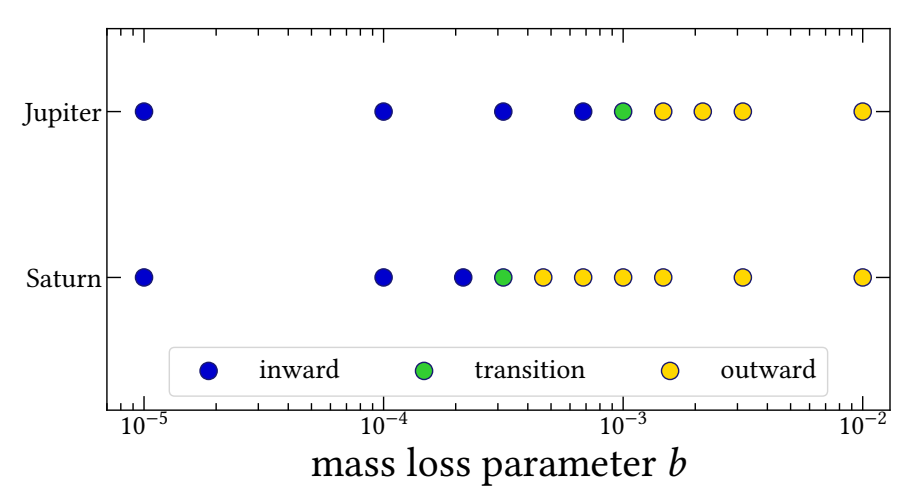

Fig. 8. Direction of migration in simulations with different mass-loss parameters $b$ for both planet masses. The lever arm is $\lambda=2.25$ in all simulations. For the models marked as transition, no clear inward or outward migration was observed.

We also experimented with different values for the magnetic lever arm while we kept the mass-loss parameter at $b=10^{-4}$, as shown in Fig. 9. The torque on the disk is proportional to $b(\lambda-1)$, so that the effect of increasing $\lambda$ is expected to be similar to that of increasing $b$. However, increasing $\lambda$ does not increase the wind mass-loss rate $\dot{\Sigma}_{\text {wind }}$, while increasing $b$ does.
Some differences in the results are therefore expected. In these simulations, we observe inward migration for low lever arms $\lambda=2.25,9$ and outward migration for high lever arms $\lambda=36,81$. As in the investigation of the $b$ parameter, the planet migrates inward for a low wind strength, and it migrates outward for high wind strengths.

\section{Discussion}

\subsection{Interpretation of the results}

Our models show that for low values of $b$ (weak wind loss) the planet migrates inward, and for high values of $b$ (strong wind loss) the planet migrates outward. In the low $b$ case the planet opens a gap and clears the co-orbital region of its gas (see, e.g., Kley \& Nelson 2012; Kanagawa et al. 2018). Therefore, no corotation torques act on the planet and it experiences only the Lindblad torques. The migration is inward and to a certain extent similar to the standard type II viscous migration, in the sense that gap formation plays a key role in the migration process. We therefore call it type IIw migration. The torque by the magnetic wind onto the disk gas causes the disk gas to gradually move inward. Consequently, the inner and outer edges of the gap also move inward. When the planet remains in its position, the outer 


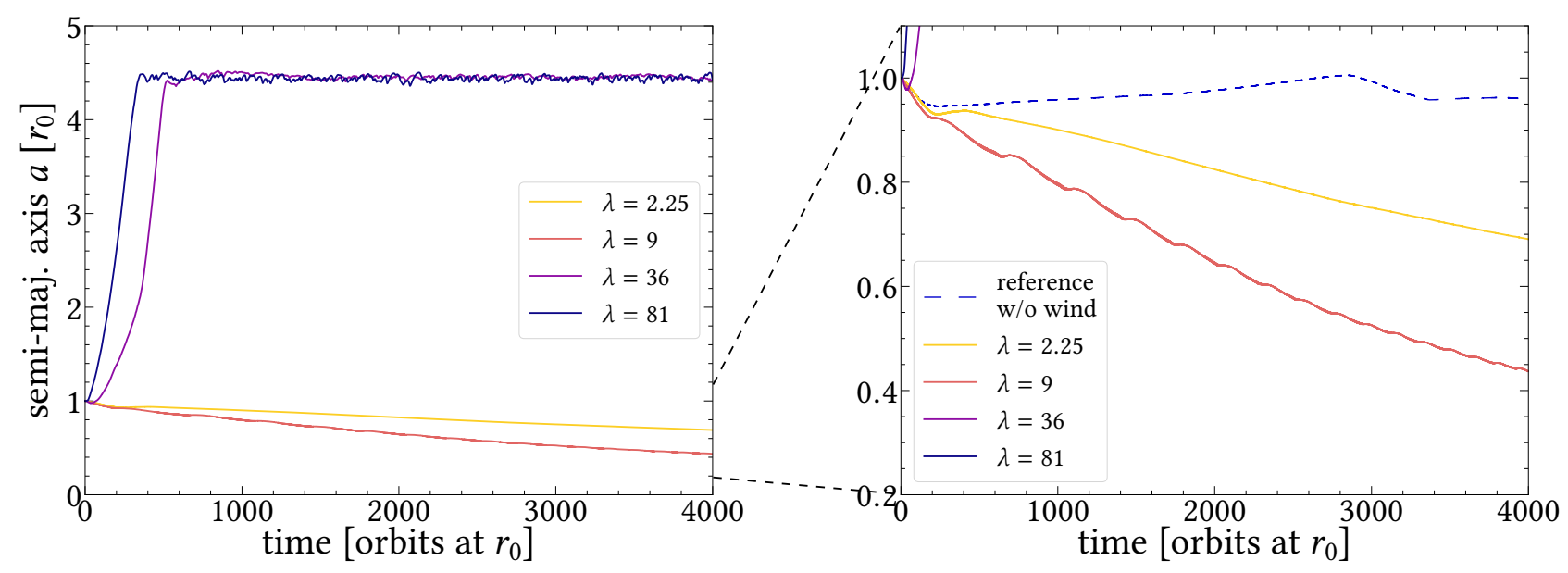

Fig. 9. Same as Fig. 7, but now varying the lever arm $\lambda$, keeping $b=10^{-4}$ in all simulations. This is studied for the Jupiter-mass planet.

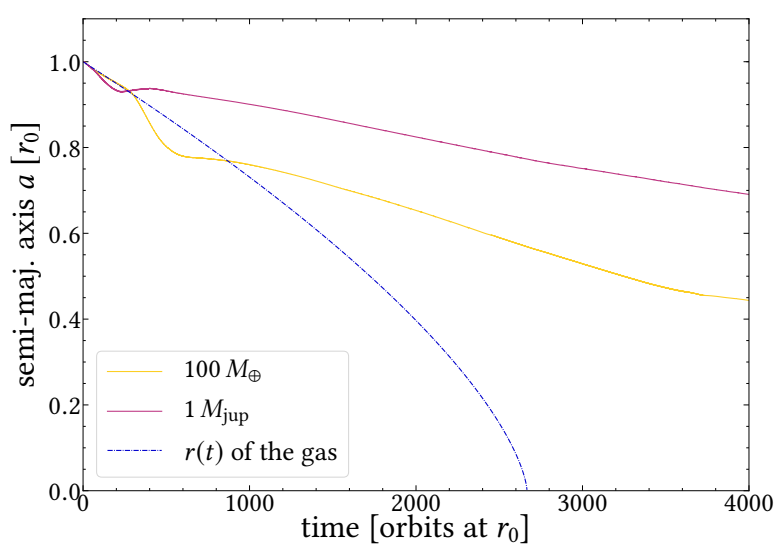

Fig. 10. Migration of the planets compared to the motion of the gas for the low $b$ case.

edge moves closer to the planet and the inner edge moves farther away from it. The Lindblad torques from the outer gap edge then dominate the Lindblad torques from the inner gap edge, and thus push the planet inward to a new equilibrium position.

If the gas drift were the only process determining the migration, the migration rate of the planet could be described by the radial velocity of the gas due to the wind $v_{\text {mig }}=v_{r}$. The planet would then be coupled to the disk and would essentially follow the disk gas as it accretes inward. A closer inspection of the results shows, however, that some gas leaks through the gap, making the type IIw migration somewhat slower than the inward motion of the gas. This is shown in Fig. 10. Similar effects are known to occur in the migration of gap-opening planets in viscous disks (Duffell et al. 2014; Dürmann \& Kley 2017).

It is interesting to note that while type IIw migration seems similar to type II migration, there is a fundamental difference between the two: in a viscous disk, the gas motion can be both inward or outward. In particular the very outer disk regions expand outward (the typical Lynden-Bell and Pringle disk evolution). This would lead to outward migration. In the case of wind-driven accretion, the wind always removes angular momentum from the disk, and therefore the gas always moves inward. Thus, type IIw also always has to point inward.

We now consider the behavior for high $b$, where the planet migrates outward at a high speed. In this case, the radial inward motion of the gas is rapid enough for it to enter the horseshoe streamlines, as shown in Fig. 11. This leads to a strong asymmetry in the horseshoe region. When gas enters the corotation region in front of the planet, it performs a horseshoe orbit and moves toward the planet. At the U-turn point of the horseshoe orbit, an excess of mass occurs. While the gas reverses its direction at this point, it moves closer to the star, which means that it loses angular momentum. This angular momentum is transferred to the planet, which in return gains it.

Behind the planet, a deficit of mass occurs because the horseshoe orbit transports the gas away from the planet. A key to maintaining this deficit is that the gas that performed the U-turn in front of the planet now lies close to the inner edge of the gap. The rapid inward motion caused by the wind then causes this gas to quickly leave the co-orbital region again by entering the inner disk. This means that this gas will not librate all the way to the far side of the planet, leaving this region devoid of gas. In the terminology of standard migration theory, this process avoids saturation of the corotation torque.

Because only the U-turn in front of the planet is populated with gas, the planet only gains angular momentum and does not lose it. The planet thus migrates outward. This outwardmigration mechanism is very similar to type III "runaway migration" (Masset \& Papaloizou 2003). In our case, however, the migration is always outward, while in the case of type III migration, the direction of the initial migration of the planet determines the direction of the runaway migration. We call this type IIIw migration.

We estimated the parameters for the transition from type IIw migration to type IIIw by comparing the libration timescale $\tau_{\text {lib }}$, which is the time it takes for a gas parcel to complete a horseshoe orbit, to the time it takes to radially cross the horseshoe region driven by the magnetic wind, which we call passing time $\tau_{\text {pass }}$. The libration timescale is (Kley \& Nelson 2012)

$\tau_{\text {lib }}=\frac{8 \pi}{3 \Omega_{\mathrm{K}, \mathrm{p}}} \frac{r_{\mathrm{p}}}{\Delta r}$,

where $r_{\mathrm{p}}$ is the distance from the planet to the star, $\Omega_{\mathrm{K}, \mathrm{p}}$ is the Kepler frequency at $r_{\mathrm{p}}$, and $\Delta r$ is the half-width of the horseshoe region. We can estimate the passing time as

$\tau_{\text {pass }}=\frac{2 \Delta r}{\left|v_{r}\left(r_{\mathrm{p}}\right)\right|}=\frac{2 \pi}{\Omega_{\mathrm{K}, \mathrm{p}} b(\lambda-1)} \frac{\Delta r}{r_{\mathrm{p}}}$, 


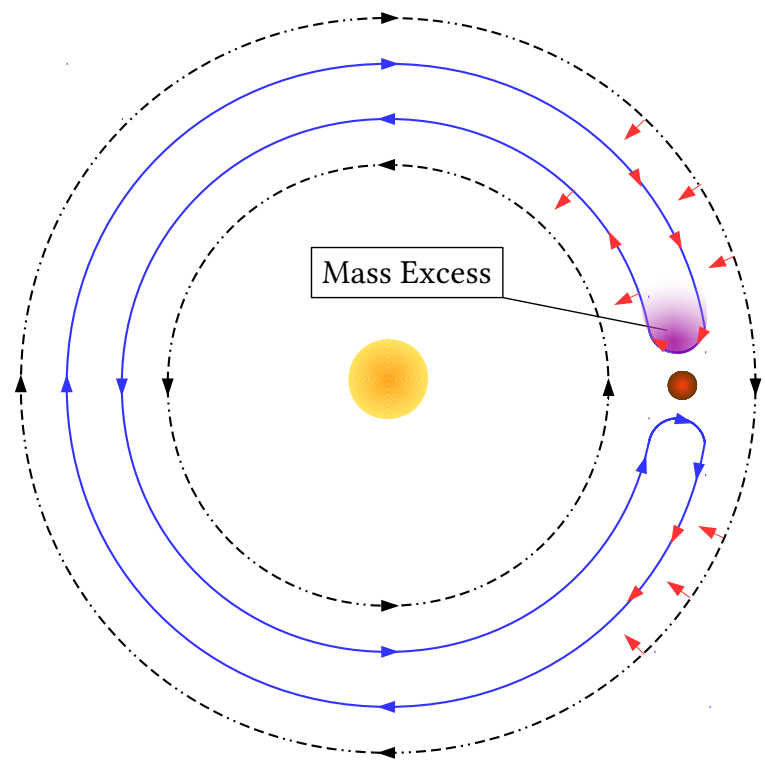

Fig. 11. Cartoon showing that the wind-driven inward motion of the gas through the co-orbital region leads to a strongly asymmetric mass distribution, with a mass excess in front of the planet and a mass deficit trailing the planet.

using Eq. (4). A comparison of the timescales results in

$$
\frac{\tau_{\text {pass }}}{\tau_{\text {lib }}}=\frac{3}{4}\left(\frac{\Delta r}{r_{\mathrm{p}}}\right)^{2} \frac{1}{b(\lambda-1)} .
$$

A shorter passing time and a longer libration time means that the gas crosses the corotation region faster than it completes the horseshoe orbit. This keeps the corotation torque unsaturated and we expect outward migration. The half-width of the horseshoe region is found to be $\Delta r=C(\epsilon) r_{\mathrm{p}} \sqrt{q / h}$, with $C(\epsilon)$ as a factor of order unity and $\epsilon$ as the smoothing length, $q$ as the planet-to-star mass ratio, and $h$ as the scale height (see Kley \& Nelson 2012). We used this to define a criterion $K=\tau_{\text {pass }} / \tau_{\text {lib }}$ for $C(\epsilon)=1$,

$K=\frac{3}{4} \frac{q}{h} \frac{1}{b(\lambda-1)}$

In our simulations, we find that the transition regime between inward and outward migration occurs for $K \approx 10$. For $K \gtrsim 10$, we find inward migration, and for $K \lesssim 10$, we find outward migration.

\subsection{Caveats}

One caveat of our flat-disk model is that we do not treat the possible vertical stratification of the coupling of the magnetic fields to the gas. It is believed that the well-shielded midplane regions of protoplanetary disks are "dead", in the sense that magnetic fields decouple from the gas. The surface layers are likely sufficiently ionized to be coupled to the field lines. This means that the torque that the magnetocentrifugal wind exerts onto the disk is only applied to the very upper layers of the disk, not to the full vertical extent of the disk. We argue that this does not make a great difference for our model because by the conservation of angular momentum, the accretion rate driven by this mechanism only depends on the torque. The only effect of the dead midplane zone is that all of this wind-driven accretion will then have to be carried by the surface layer material. For the present model it is irrelevant whether the accretion is carried by the full column of the disk or only by the surface layers.

Furthermore, our parameterization of the winds assumed a constant lever arm and mass-loss parameter throughout the whole disk. A better approximation would be a radial dependency of both parameters. The parameters are not well known so far, therefore the values we used are only estimates.

The resolution of our models is also a problem. Especially if the mass of the planet is low, the width of the co-orbital region is narrow, requiring high spatial resolution. We have already seen the effect of resolution on the results in Sect. 4, when we discussed the case of $1 M_{\mathrm{Jup}}$ and $b=10^{-3}$.

The strongest caveat of our simplified approach is that our model does not set the wind rate self-consistently. The wind rate and lever arm, and thereby the torque onto the disk, have to be completely parameterized. Improvement requires a detailed 3D magnetohydrodynamic simulation of the driving of the wind. Such models exist (see, e.g., Béthune et al. 2017; Wang \& Goodman 2017; Wang et al. 2019), but they are costly. To calculate the effect on planetary migration, many thousand orbits have to be computed, which is a challenge for such models. Nevertheless, this is the path that has to be taken in the future.

\subsection{Comparison to other work}

The effect of wind mass loss of the disk on type I migration has been studied before, for example, by Ogihara et al. (2015). In this study, however, the main effect was the change of the radial profile of the disk surface density $\Sigma(r)$, which, using the standard type I migration rate formula (e.g., Paardekooper et al. 2011), leads to a modified migration rate. In contrast, in our paper we did not study how the changed $\Sigma(r)$ profile affects the migration rate (although it is, in a way, obtained for free), but instead focused on the computation of the torque itself.

In addition to wind-driven accretion as a replacement for the classic $\alpha$-viscosity model, there may be other drivers of accretion. For instance, McNally et al. (2017) showed that the presence of a vertical magnetic field in the dead zone of a disk could lead to the formation of strong spiral-shaped magnetic fields in the plane of the disk through the Hall effect. These fields transport angular momentum within the disk and lead to accretion, even though the disk is laminar. The authors called this situation a magnetically torqued dead zone. When a planet is inserted into such a disk, this can lead to similar asymmetric filling or depletion of the co-orbital region and to the corresponding type-III-like migration effects as we find in our paper (McNally et al. 2017, 2018). In our model, we need a vertical magnetic field to drive a wind, therefore it is very well possible that both effects act simultaneously.

\section{Conclusions}

Our models show that the effect of magnetocentrifugal winddriven accretion on planet migration can be profound. For very strong winds, it can even lead to rapid outward migration similar to type III migration (which we call type IIIw migration). In this case, however, the direction of migration is deterministic: it does not depend on an initial "seed motion" of the planet. The parameter range for which we find that rapid outward migration may occur is coupled to a rapid evolution of the disk, however. We find that lower mass planets are more prone to the type IIIw effect and therefore more easily migrate outward. We speculate that this type IIIw outward migration mechanism may 


\section{N. Kimmig et al.: Winds and migration}

be a possible origin of the intermediate-mass planets at large radii that are thought to be the cause of the multi-ringed ALMA disks (Huang et al. 2018; Zhang et al. 2018). These planets would then have formed at much smaller semi-major axes and then been efficiently transported outward to their final location.

Acknowledgements. We thank Leonardo Krapp, Philipp Weber and Thomas Rometsch for assistance with the required modifications to FARGO3D, and Hubert Klahr for useful comments. We also thank the referee, John Chambers, for his constructive and helpful comments that substantially improved the paper. Part of this work was supported by DFG grant DU 414/18-1 and KL 650/27-1 within the Priority Programme "Exploring the Diversity of Extrasolar Planets" (SPP 1992) and DFG research group FOR 2634 "Planet Formation Witnesses and Probes: Transition Disks" under grant DU 414/23-1 and KL 650/30-1. For the simulations performed on the BwForCluster BinAC, we also acknowledge support by the High Performance and Cloud Computing Group at the Zentrum für Datenverarbeitung of the University of Tübingen, the state of Baden-Württemberg through bwHPC and the German Research Foundation (DFG) through grant no INST 37/935-1 FUGG.

\section{References}

Ataiee, S., Pinilla, P., Zsom, A., et al. 2013, A\&A, 553, L3

Bae, J., Zhu, Z., \& Hartmann, L. 2017, ApJ, 850, 201

Bai, X.-N. 2016, ApJ, 821, 80

Bai, X.-N., \& Stone, J. M. 2013, ApJ, 769, 76

Baruteau, C., \& Masset, F. 2008, ApJ, 672, 1054

Benítez-Llambay, P., \& Masset, F. S. 2016, ApJS, 223, 11

Béthune, W., Lesur, G., \& Ferreira, J. 2017, A\&A, 600, A75

Blandford, R. D., \& Payne, D. G. 1982, MNRAS, 199, 883

Dong, R., Li, S., Chiang, E., \& Li, H. 2017, ApJ, 843, 127

Duffell, P. C., Haiman, Z., MacFadyen, A. I., D’Orazio, D. J., \& Farris, B. D. 2014, ApJ, 792, L10
Dürmann, C., \& Kley, W. 2017, A\&A, 598, A80

Ferreira, J. 2008, New Astron. Rev., 52, 42

Flaherty, K. M., Hughes, A. M., Rosenfeld, K. A., et al. 2015, ApJ, 813, 99

Flaherty, K. M., Hughes, A. M., Teague, R., et al. 2018, ApJ, 856, 117

Gammie, C. F. 1996, ApJ, 457, 355

Hasegawa, Y., Okuzumi, S., Flock, M., \& Turner, N. J. 2017, ApJ, 845, 31

Huang, J., Andrews, S. M., Dullemond, C. P., et al. 2018, ApJ, 869, L42

Kanagawa, K. D., Tanaka, H., \& Szuszkiewicz, E. 2018, ApJ, 861, 140

Kley, W., \& Crida, A. 2008, A\&A, 487, L9

Kley, W., \& Nelson, R. P. 2012, ARA\&A, 50, 211

Koller, J., Li, H., \& Lin, D. N. C. 2003, ApJ, 596, L91

Li, H., Finn, J. M., Lovelace, R. V. E., \& Colgate, S. A. 2000, ApJ, 533, 1023

Li, H., Lubow, S. H., Li, S., \& Lin, D. N. C. 2009, ApJ, 690, L52

Masset, F. S. 2001, ApJ, 558, 453

Masset, F. S., \& Papaloizou, J. C. B. 2003, ApJ, 588, 494

McNally, C. P., Nelson, R. P., Paardekooper, S.-J., Gressel, O., \& Lyra, W. 2017, MNRAS, 472, 1565

McNally, C. P., Nelson, R. P., \& Paardekooper, S.-J. 2018, MNRAS, 477, 4596

Ogihara, M., Morbidelli, A., \& Guillot, T. 2015, A\&A, 584, L1

Paardekooper, S.-J., Baruteau, C., \& Kley, W. 2011, MNRAS, 410, 293

Pinte, C., Dent, W. R. F., Ménard, F., et al. 2016, ApJ, 816, 25

Rafikov, R. R. 2002, ApJ, 572, 566

Shakura, N. I., \& Sunyaev, R. A. 1973, A\&A, 24, 337

Simon, J. B., Bai, X.-N., Armitage, P. J., Stone, J. M., \& Beckwith, K. 2013, ApJ, 775,73

Spruit, H. C. 1996, ArXiv e-prints [arXiv:astro-ph/9602022]

Suzuki, T. K., Ogihara, M., Morbidelli, A., Crida, A., \& Guillot, T. 2016, A\&A, 596, A74

Tanaka, H., Takeuchi, T., \& Ward, W. R. 2002, ApJ, 565, 1257

Wang, L., \& Goodman, J. J. 2017, ApJ, 835, 59

Wang, L., Bai, X.-N., \& Goodman, J. 2019, ApJ, 874, 90

Ward, W. R. 1997, Icarus, 126, 261

Yu, C., Li, H., Li, S., Lubow, S. H., \& Lin, D. N. C. 2010, ApJ, 712, 198

Zhang, S., Zhu, Z., Huang, J., et al. 2018, ApJ, 869, L47 